\title{
A Theoretical Model of the Tridimensional Structure of Bacillus thuringiensis subsp. medellin Cry 11Bb Toxin Deduced by Homology Modelling
}

\author{
Pablo Gutierrez/ ${ }^{++}$Oscar Alzate*/+++, Sergio Orduz $/^{+}$
}

Unidad de Biotecnología y Control Biológico, Corporación para Investigaciones Biológicas, AA7378, Medellin, Colombia *Universidad Pontificia Bolivariana, Medellin, Colombia

Cryl $1 \mathrm{Bb}$ is an insecticidal crystal protein produced by Bacillus thuringiensis subsp. medellin during its stationary phase; this $\partial$-endotoxin is active against dipteran insects and has great potential for mosquito borne disease control. Here, we report the first theoretical model of the tridimensional structure of a Cryl1 toxin. The tridimensional structure of the Cryl1Bb toxin was obtained by homology modelling on the structures of the CrylAa and Cry3Aa toxins. In this work we give a brief description of our model and hypothesize the residues of the CryllBb toxin that could be important in receptor recognition and pore formation. This model will serve as a starting point for the design of mutagenesis experiments aimed to the improvement of toxicity, and to provide a new tool for the elucidation of the mechanism of action of these mosquitocidal proteins.

Key words: homology modelling - $\partial$-endotoxin structure - Cry proteins - Bacillus thuringiensis

Bacillus thuringiensis is a Gram-positive endospore forming bacterium characterized by the production of parasporal crystalline proteic inclusions (which contain $\partial$-endotoxins) during the stationary phase. $\partial$-endotoxins are highly toxic and specific to insects of the Coleopteran, Lepidopteran and Dipteran orders (Schnepf et al. 1998), and once in the insect midgut, they are activated by gut proteases, followed by binding to specific receptors on the cells lining the larval midgut (Hofmann et al. 1988). This interaction promotes their insertion into the membrane, forming ion selective channels by oligomerization of toxin monomers (Gazit \& Shai 1993, Aronson et al. 1999), and the insect dies by loss of osmotic pressure regulation (Knowles \& Ellar 1987).

$\partial$-endotoxins, also known as Cry proteins, are classified according to their degree of evolutionary divergence into 22 groups (Crickmore et al.

This work received financial support from Colciencias, and Corporación para Investigaciones Biológicas, Medellin, Colombia.

${ }^{+}$Corresponding author. Fax: +(574)-441-5514. E-mail: sorduz@epm.net.co

${ }^{++}$Current address: Department of Biochemistry, McGill University, Montreal, Canada

${ }^{+++}$Current address: Biochemistry Department, The Ohio State University, 484 W 12th Ave., OH 43210, USA

Received 15 May 2000

Accepted 25 August 2000
1998). The Cry 11 family of $\partial$-endotoxins is comprised of dipteran-active proteins where the Cry11Aa protein of B. thuringiensis subsp. israelensis has been the most intensively studied. At present, there is interest in identifying new dipteran-active toxins for their importance in mosquito and black fly control (Orduz et al. 1992, Ragni et al. 1996). Mosquitocidal activity has also been found in Cry1 Ab, Cry1Ca, Cry2Aa, Cry11Ba, Cry11Aa, Cry16Aa, Cry19Ba, Cyt1Aa, Cyt1Ab, Cyt2Aa and some Cry-related proteins (Cry 17Aa, Cry18Aa and Cry 19Aa) produced by Clostridium bifermentans subsp. malaysia (Schnepf et al. 1998).

To date, the structures of two Cry proteins, Cry1 Aa and Cry3Aa have been reported ( $\mathrm{Li}$ et al. 1991, Grochulski et al. 1995). They are composed of three domains, and the high structural similarity between them, despite the low aminoacid homology, suggests the conservation of many structural features among $\partial$-endotoxins. In these Cry proteins, domain I consists of seven alpha helices in which helix 5 is surrounded by the others, forming a helical bundle. Several studies have shown that this domain is responsible for channel ion formation (Walters et al. 1993, von Tersch et al. 1994). Domain II consists of three antiparallel B-sheets joined in a greek key topology, arranged in a Bprism, and its function is associated with receptor recognition and binding (Schnepf et al. 1990, Gill et al. 1992, Knowles 1994, Lu et al. 1994). Domain III is formed by two antiparallel $\beta$-sheets forming a $\beta$-sandwich in a jelly roll topology; the role of this domain is unclear but it seems impor- 
tant for insect specifity and protein stability (Bosch et al. 1994, Masson et al. 1994, Lee et al. 1999).

B. thuringiensis subsp. medellin is a potentially important strain for mosquito control (Orduz et al. 1992, Ragni et al. 1996, Thiéry et al. 1998). Sodium dodecyl sulfate polyacrylamide gel electrophoresis (SDS-PAGE) analysis of the $B$. tharingiensis subsp. medellin parasporal inclusion indicates that this strain produces a polypeptide of $94 \mathrm{kDa}$, multiple bands between 80 and $65 \mathrm{kDa}$, and two doublets at 40-41 and 28-30 kDa (Orduz et al. 1994). The $94 \mathrm{kDa}$ protein (Cry11Bb) has been cloned into Escherichia coli and B. thuringiensis and was shown to be responsible for most of the mosquitocidal activity. The mode of action of Cry11 $\partial$-endotoxins is still not clear, and though a series of studies have been made (Dai \& Gill 1993, Feldmann et al. 1995, Orduz et al. 1998, Thiéry et al. 1998), no structural information is still available.

In this work, we propose a model for the structure of the Cry $11 \mathrm{Bb} \partial$-endotoxin based on the hypotheses of structural similarity with Cry1 Aa and Cry3Aa toxins. This model provides a starting point for the design of mutagenesis experiments aimed to elucidate the mechanism of action of the Cry11 family of toxins.

\section{MATERIALS AND METHODS}

Sequence alignment between Cry $11 \mathrm{Bb}$ (Genbank accession AAC97162, Orduz et al. 1998), Cry1 Aa and Cry3Aa (PDB entries 1CIY and 1DLC respectively), was generated using the structural alignment tool of the program SwissPdbViewer (Guex \& Peitsch 1997) and corrected manually until a satisfactory placement of conserved blocks and aminoacid identities was obtained. This alignment was submitted to SwissModel in the expasy server (http://www.expasy.ch/ spdbv/) and a preliminary model for Cry $11 \mathrm{Bb}$ was retrieved. Loops and side chains conformations were recalculated with the OPLS force field without distance restrains of the Hyperchem program (Hypercube, Inc.) and the most severe steric overlaps removed. The model was validated with PROCHECK (Laskowski et al. 1993) and WHAT IF (Vriend 1990) programs by submitting the coordinates to the EMBL server (http://www.emblheidelberg.de/). Sequence identities were calculated with Needleman and Wunsch maximum matching algorithm of the MacDNASIS program (Hitachi, Software). Figures, electrostatic potentials, and Ca RMSD calculations were generated with SwissPdbViewer (Guex \& Peitsch 1997).

\section{RESULTS}

Based on the structural alignment of the amionoacid sequence of the Cry $11 \mathrm{Bb}$ toxin with
Cry1Aa and Cry3Aa toxins (Fig. 1) a theoretical model of the Cry $11 \mathrm{Bb}$ toxin was obtained, and corresponds to residues $15-620$ of the primary structure (Fig. 2). Alignment of domain I was straightforward and the highly conserved nature of helix 5 in the Cry $11 \mathrm{Bb}$ toxin made placement of the other residues in this domain reliable. According to genetic studies and the structural features observed in Cry1Aa and Cry3Aa toxins, large insertions and deletions seem unlikely to be present here. Alignment of domain II was less reliable and had to be corrected manually. The limits of this domain could be determined because of the conserved nature of flanking domains I and III. Placements of large insertions or deletions were mostly done in regions corresponding to the loops connecting $\beta$ sheets involved in receptor recognition, therefore the most variable region of the molecule, and their lengths were determined by selecting the combination that allowed the best conservative profile of the neighboring aminoacids. Domain III is quite well conserved on the $\mathrm{N}$-terminal side, but on the C-terminal side, there was no clear correspondence of aminoacids (only one identity present at Ile114) making difficult to define the end of the molecule.

Structural comparison of the Cry $1 \mathrm{Aa}, \mathrm{Cry} 3 \mathrm{Aa}$ toxins with the theoretical model of the Cry $11 \mathrm{Bb}$ protein indicates correspondence to the general model for a Cry protein, and the few differences found were located in the loops of domains II and III (Fig. 3). The superimposed backbone traces of Cry1Aa and Cry3Aa displayed 0.66 and $0.83 \AA$ RMS deviations for $\mathrm{C} \alpha$ (Fig. 4). The Ramachandran plot (data not shown) indicated that most (95\%) of residues have $\phi$ and $\psi$ angles in the core and allowed regions, except for some proline and glycine residues and few residues located in the loop regions. Most bond lengths, bond angles, and torsion angles were in the range of values expected for a naturally folded protein (data not shown). The structural model shown in Fig. 2 indicates that it contains all the general features of the Cry toxins (an $\alpha+\beta$ structure with three domains). Four of the five Cys residues present in the primary structure were included in the Cry11Bb model (Cys157, Cys186, Cys225 and Cys436), but it seems that they are not involved in structure stabilization through disulfide bonds, since the shortest S-S distance (Cys157-Cys225) between the six different possible bonds was $18.51 \AA$.

\section{DISCUSSION}

Domain I - Domain I is composed of residues 15-256 and consists of $9 \alpha$-helices and two small $\beta$-strands. The identified helices and strands are: $\alpha 1$ (Leu19-Leu32); $\alpha 2$ a (Ala39-Gln53); $\alpha 2 b$ (Ile62-Lys73); $\alpha 3$ (Gln79-Phe108); $\alpha 4$ (Phe117- 


\begin{abstract}
Cry11Bb 15 SSFPLFNSKI EPSIAPALIA VAPIAKYLAT ALAKWALKQG FAKLKSEIFP GNETATMEKV RLEVQTILNQ Cry3Aa 61 TTKDVIQKGI SVVGDLLGVV GFPFGGALVS FYTNFLNTIW -PSE-DPWKA FMEQVEALMD QKIADYAKNK Cry1Aa 33 YTPIDISL SLTQFLLSEF VPGAG--FVL GLVDI---IW GIFGPSQWDA FLVQIEQLIN QRIEEFARNQ

CrY11Bb 85 TLQTDRVATL KAEYEGFIHL GKVFTDYVSQ STFTPATAKT HFLNMSNLLI QRLPQFEIAG YEGVSISLFT CrY3Aa 129 ALAELQGLQN NVEDYVSALS SWQKNPVSSR NPHSQGRIRE LFSQAESHFR NSMPSFAISG YEVLFLTTYA CrY1Aa 96 AISRLEGLSN LYQIYAESFR EWEAD--PTN P-ALREEMRI QFNDMNSALT TAIPLLAVQN YQVPLLSVYV

Cry11Bb 155 QMCTLHLGLL KDGILAGSDW GFTPEDKDSL ICQFNRYVNE YNTRMMGLÝ IEFGRLLAKN LNEALNFRNM CrY3Aa 199 QAANTHLFLL KDAQIYGEEW GYEKEDIAEF YKRQLKLTQE YTDHCVKWYN VGLDKLRGSS YESWVNFNRY CrY1Aa 164 QAANLHLSVL RDVSVFGQRW GFDAATINSR YNDLTRLIGN YTDYAVRWYN TGLERVWGPD SRDWVRYNQF $\star \quad \star * . * . *$. * * * .

Cry11Bb 225 CSLYVFPFSE AWYLLRYEGT KLENTLSLWN FVGEDIGGIL HNDWKGALYK LLMGATNQRL ANVRFNYSYF Cry3Aa 269 RREMTLTVLD LIALFPLYDV RLYPKEVKTE LTRDVLTDPI VG--VNNLRG YGTTFSNIEN YIRKPHLFDY Cry1Aa 234 RRELTLTVLD IVALFSNYDS RRYPIRTVSQ LTREIYT-NP VLENFDGSF- -RGMAQRIEQ NIRQPHLMDI

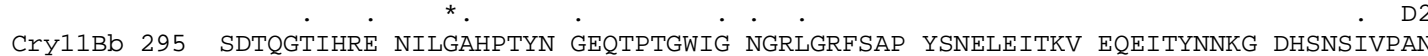
CrY3Aa 337 LHRIQFHTRF QPG-----YY GNDSFNYWSG NYVSTRPSIG -SND-IITSP FYGN------ ---KSSEPVCry1Aa 300 LNSITIYTDV HR------- ---GFNYWSG HQITASPVGF SGPE--FAFP LFGN------ ---AGNAAPP

CrY11Bb 365 TRNEILTATV PITADPFFKT ADIN-_--CrY3Aa 390 QNLEFN-GEK VYRAVANTNL AVWP------ SAVYSGVTKV EFSQYNDQTD EASTQTYDSK RNVGAVSWDS Cry1Aa 348 VLVSLT-GLG IFRTLSSPLY RRIILGSGPN NQELFVLDGT EFSFASLT-T NLPSTIYRQ- ----RGTVDS

CrY11Bb 420 NRLEYDGYYI RAVSACPRNV PLSYNHNYLT LTYNRLEYDA PTTQNIIVGF SPNNTKSFYA RNSHYLSATN CrY3Aa 453 IDQLP-PETT DEPLEKGYSH QLNYVMCFLM QGS---RGTI PVLTWTHKSV D----FFNMI DSKKITQLPI Cry1Aa 411 LDVIP-PQDN SVPPRAGFSH RLSHVTMLSQ AAGAVYTLRA PTFSWQHRSA E----FNNII PSSQITQIPL

Cry11Bb 491 DAYVIPALQF ATVSDRSFLE DTPDQATDGS IKFTETVLGN CrY3Aa 515 VKAYKLQSGA SVVAGPRFTG GDIIQCTENG SAATIYVTPD VSYSQKYRAR IHYASTSQIT FTLSLDGAPF Cry1Aa 476 TKSTNLGSG SVVKGPGFTG GDILRRTSPG QISTLRVNIT APLSQRYRVR IRYASTTNLQ FHTSIDGRPI

Cry11Bb 558 ARLAAGIRVR SQNSGNNRLL GGIPVEGNSG WVDYITDSFT FNDLGITTAS TNAFFSIDSD GVN CrY3Aa 585 NQYYFDKTIN K-GDTLTYNS FNLASFSTPF ELSG--NNLQ IGVTGLSAGD KVYIDKIEFI PVN Cry1Aa 546 NQGNFSATMS S-GSNLQSGS FRTVGFTTPF NFSNGSSVFT LSAHVFNSGN EVYIDRIEFV PAEVT
\end{abstract}

Fig. 1: sequence and structural alignment of Cry1 Aa, Cry3 Aa and Cry11Bb. * denotes identical residues, the point indicates conserved residues, D2 and D3, the initiation of domains 2 and 3 respectively.
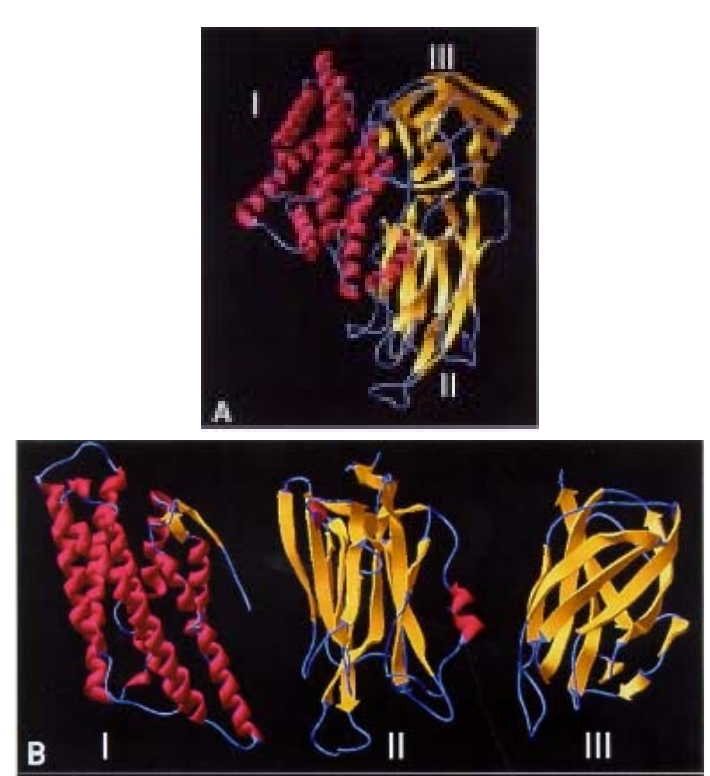

Fig. 2: homology model deduced for the tridimensional structure of the Cry11Bb toxin. A: domain I is mostly alpha helical (fuchsia), specificity domain II is located in the middle and domain III on the right side; B: from left to right domains I, II and III
Pro138); $\alpha 5$ (Ser151-Ile168); $\alpha 6$ (Pro178Arg209); $\alpha 7$ a (Leu215-Phe230); $\alpha 7 b$ (Glu234Tyr241); B0 (Ala34-Ala36) and B1a (Thr249Leu252). All the helices in the Cry11Bb model were slightly shorter than those in Cry1Aa and Cry3Aa, and less amphiphilic (Table I). According to the amphiphilicity calculated with the Hoops and Woods values, the most exposed helices are $\alpha 1, \alpha 2 \mathrm{a}, \alpha 2 \mathrm{~b}, \alpha 3$ and $\alpha 6$, which corresponds well with the accessibility calculated with SwissPDB, except for $\alpha 1$, which is packed against domain II. It is possible that this helix will have some mobility, if we take into consideration that one of the cutting sites by gut proteases is located between Ser56 and Ile58, close to the middle of this helix (Segura et al. 2000). The charge distribution pattern in the Cry $11 \mathrm{Bb}$ theoretical model corresponds to a negatively charged patch along $B 4$ and $B 13$ of domains II and III respectively.

The Cry $11 \mathrm{Bb}$ domain I model correlates well with data from Gazzit et al. (1998) who suggested that $\alpha 4$ and $\alpha 5$ insert into the membrane in an antiparallel manner as an helical hairpin. It is possible that according to the surface electrostatic po- 
tential of helices 4 and 5 (Fig. 5), there was a neutral region in the middle of the helices which probably indicates, if the umbrella model is correct, that both helices cross the membrane with their polar sides exposed to the solvent, as it has been suggested by the results of mutagenesis experiments done by Kumar and Aronson (1999) with the Cry1Ac toxin. This region is also the most conserved among the Cry toxins.

Kumar and Aronson (1999) demonstrated that mutations in the base of helix 3 and the loop between $\alpha 3$ and $\alpha 4$ that cause alterations on the bal- ance of negative charged residues can cause loss of toxicity by Cry1Ac. Mutations in helices $\alpha 2$, $\alpha 6$ and the surface residues of $\alpha 3$ have no important effect on toxicity; meanwhile, helices $\alpha 4$ and $\alpha 5$ seem to be very sensitive to mutations. Helix $\alpha 1$ probably does not play an important part in toxin activity after cleavage of the protoxin. It is possible that mutations aimed to an increase in amphilicity in these helices will improve the pore forming activity of Cry11 type of toxins.

Domain II - This is the most variable domain among Cry toxins, and it has been shown that it is

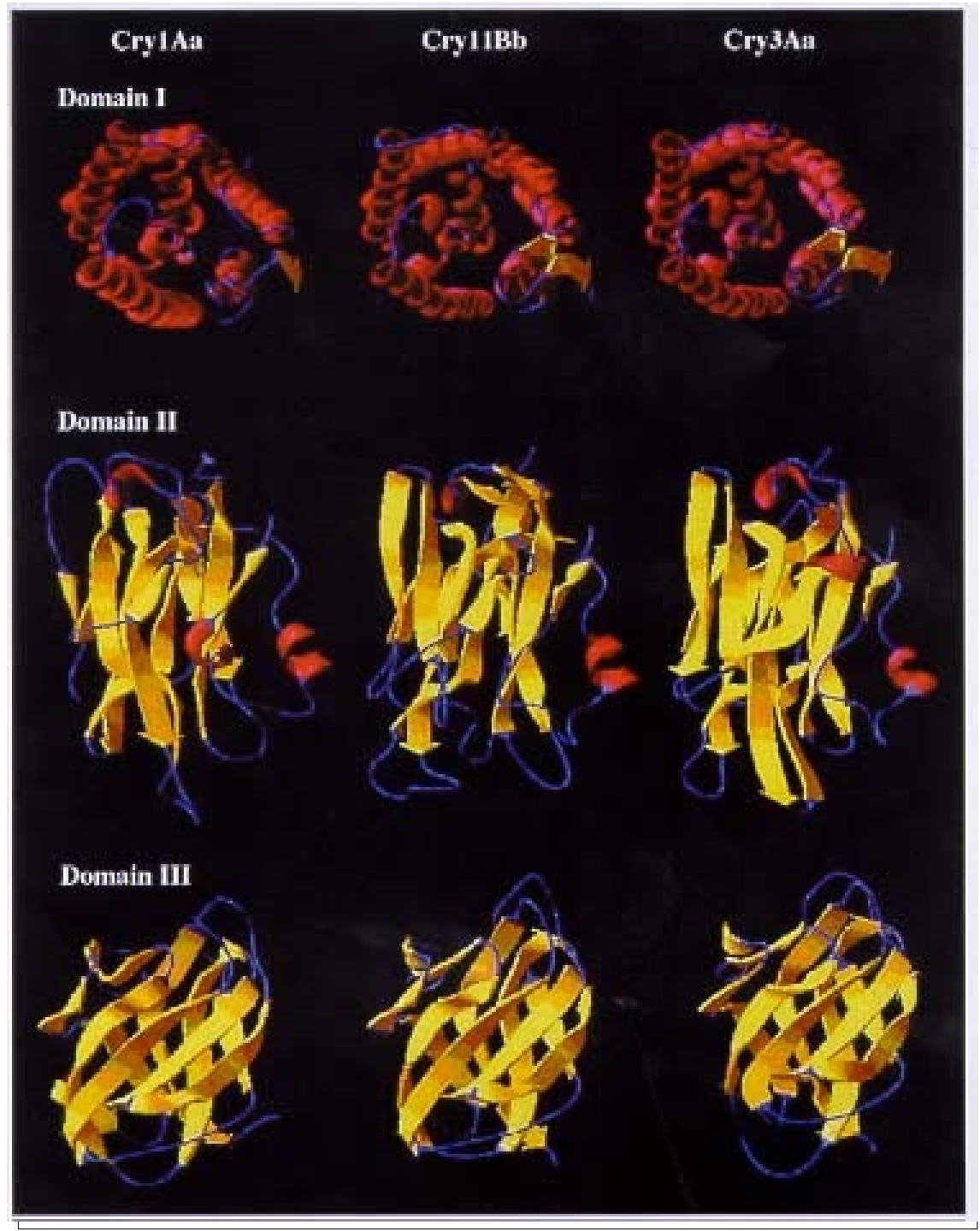

Fig. 3: comparison of the structural domains of the Cry1Aa, Cry11Bb, and Cry3A. Upper view of domain I shows that the three toxins are almost identical. Front view of domain II where most of the differences among the three toxins are located, specifically in the loops at the bottom of the figure, and probably due to the presence of specificity determinants which recognize distinct receptors in the insect midgut cells. Front view of domain III which does not show major differences except for loop $\beta 16-\beta 17$, located at the bottom of the figure. 


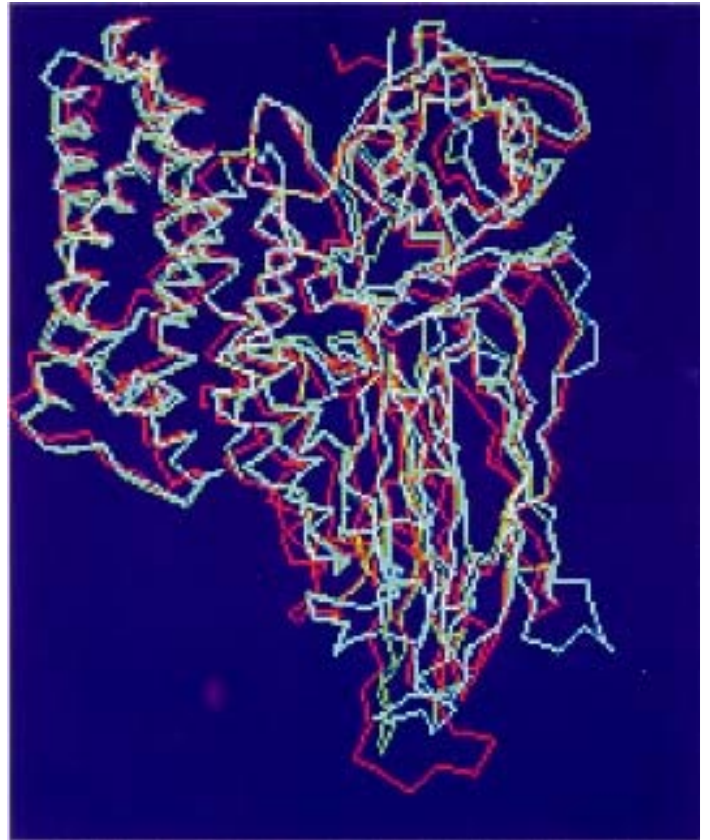

Fig. 4: superposition of the $\alpha \mathrm{C}$ carbon traces of Cry $1 \mathrm{Aa}$ (blue), Cry3 Aa (green) and Cry11Bb (red) toxins of Bacillus thuringiensis. The main differences in the three toxins are located in loops of domain II, which is implicated in receptor recognition and binding.

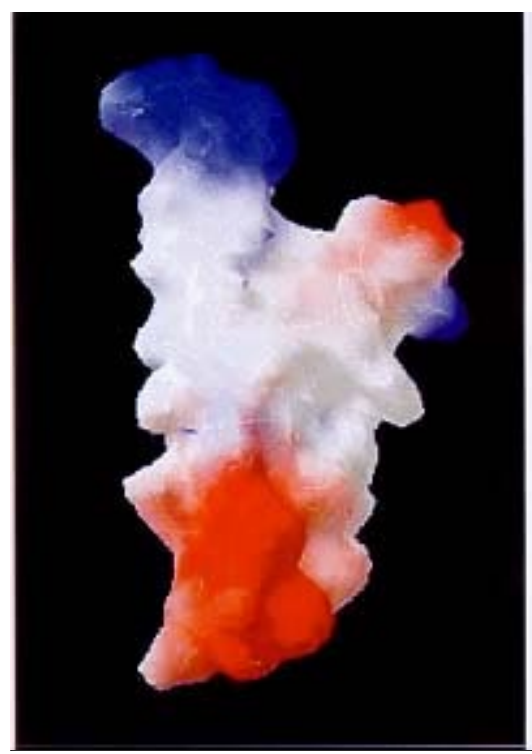

Fig. 5: surface electrostatic potential of helices 4 and 5 located in domain I of the Cry $11 \mathrm{Bb}$ toxin of Bacillus thuringiensis subsp. medellin showing the charged and neutral surfaces of the extremes and middle portions; this supports the notion that these helices are important in membrane insertion. Negative and positive regions are red and blue, respectively. involved in receptor recognition and therefore considered as the specificity determining region. As for other Cry toxins, domain II of the Cry $11 \mathrm{Bb}$ toxin consists of three greek key beta sheets arranged in a beta prism topology. It is comprised by residues $257-478$, one helix ( $\alpha 8$, Ala279Ala285) and 11 B-strands (B2 Ser292-Asn305, B3 Pro319-Ser332, B4 Ile341-Lys343, B5 Thr365Iso369, B6 Val374-Phe381, ß7 Trp389-Leu396, 38 Asn401-Arg407, $\beta 9$ Ile418-420, ß10 Pro437Thr450, 311 Tyr458-Val468 and $B 12$ Phe470Lys476). The main difference between the Cry $11 \mathrm{Bb}$ theoretical model and Cry $1 \mathrm{Aa}$ and Cry3Aa structures is the length of the two loops joining the apical $\beta$-strands ( $\beta 2-\beta 3$ and $\beta 4-\beta 5$ ) located between L307-Y313 and I348-N358. We propose that these residues are implicated in receptor binding, and consequently in specificity of the Cry $11 \mathrm{Bb}$ toxin. The first insertion between strands B2 and B3, the N-terminal part of this loop (Ile306Thr311) is mostly hydrophobic, while the C-terminal half (Thr312-Thr318) is polar and have one positively charged residue (Glu316). This loop probably interacts with the receptor through both hydrophobic and electrostatic interactions; Gly315 probably helps in receptor binding by providing more mobility to Glu316 that may interact through salt bridges with the receptor. Loop $\$ 4-\$ 5$ is mostly hydrophilic, and the charged residues located at the tip of the loop (Lys353, Asp355 and His356) are probably important determinants of insect specificity. As in loop $\beta 2-\beta 3$, a glycine residue (354) is also present before a negatively charged residue (Asp355) supporting the hypothesis that correct orientation of charged residues in the specificity loops could be important in receptor recognition. The third insertion (Ser471Lys476) probably does not play an important role in insect recognition as it is located in the region joining domains II and III, but it probably gives more mobility to domain III modulating its function in receptor binding.

Mutations in defined regions of the Cry1Aa toxin have identified residues 365-371 (equivalent to residues in the Cry11 Bbß6-B7 loop), as essential for binding to the membrane of midgut cells of Bombyx mori (Ge et al. 1989, Lu et al. 1994). In the Cry $11 \mathrm{Bb}$ model, this region is shorter than their counterparts in Cry1Aa and Cry3Aa. Loop ß2-B3 seems also to be able to modulate the toxicity and specificity of Cry1C (Smith \& Ellar 1994), in this region it was found a five residue insertion (Gly308-Thr312). The dual specificity of Cry2Aa for Lepidoptera and Diptera has been mapped to residues 307-382 that corresponds in the Cry $11 \mathrm{Bb}$ theoretical model to sheet 1 , strand B6, and loop B6- $\beta 7$, where most of the insertions/deletions and structural differences were located. 
TABLE

Length $(\AA)$ and hydrophobic moments of helices in domain I of Cry1Aa, Cry3Aa and Cry11Bb toxins of Bacillus thuringiensis

\begin{tabular}{lcccccccc}
\hline Toxin & $\alpha 1$ & $\alpha 2 \mathrm{a}$ & $\alpha 2 \mathrm{~b}$ & $\alpha 3$ & $\alpha 4$ & $\alpha 5$ & $\alpha 6$ & $\alpha 7 \mathrm{a}$ \\
\hline Cry11Bb & $20.21^{a}$ & 20.69 & 17.40 & 43.10 & 33.64 & 26.27 & 46.75 & 22.71 \\
& $(0.37)^{b}$ & $(0.55)$ & $(0.20)$ & $(0.25)$ & $(0.04)$ & $(0.58)$ & $(0.48)$ & $(0.14)$ \\
Cry1Aa & 18.23 & 12.22 & 14.99 & 40.08 & 34.53 & 19.75 & 46.45 & 15.32 \\
& $(0.25)$ & $(0.62)$ & $(0.67)$ & $(0.63)$ & $(0.460$ & $(0.51)$ & $(0.78)$ & $(0.51)$ \\
Cry3A & 20.80 & 20.62 & 20.12 & 43.21 & 34.93 & 26.89 & 47.06 & 22.75 \\
& $(0.96)$ & $(0.62)$ & $(1.26)$ & $(0.53)$ & $(0.58)$ & $(0.39)$ & $(1.05)$ & $(0.70)$ \\
\hline
\end{tabular}

$a$ : length in $\AA$; $b$ : hydrophobic moments in parenthesis

Domain III - This domain showed high conservation of residues and the only important modification is a 3-residue deletion between $B 16$ and $\beta 17$. The $B$-strands in this domain are $\beta 13 \mathrm{a}$ (Tyr485-Asn490), B3b(Ile495-Ala497), ß14 (Ala501-Val503), B15 (Pro513-Ala516), B16 (Ser520-Gly529), ß17 (Lys533-Asn543), ß18 (Thr546-Arg553), $B 19$ (Lys555-Ala562), ß20 (Gly579-Glu583), ß21 (Ile592-Leu601), ß22 (Thr608-Val619). We propose the sequence AKYSIRLNTGF as homolog to conserved block 4 . In the case of conserved block 5 there was only one identity and two conserved residues (Ile614, Phe611 and Asp615, respectively). Several studies indicate that site mutations in this block reduce toxicity and alter channel properties in Cry1Ac (Lee et al. 1999) and Cry1Aa (Chen et al. 1993, Schwartz et al. 1997), divergence in block 5 possibly reveals an alternative mechanism of membrane permeabilization.

In conclusion, evidences presented here, based on the identification of structural equivalent residues of Cry1Aa and Cry3Aa in Cry11Bb toxin through homology modelling indicate that, despite the low aminoacid homology among these three toxins, they share a common tridimensional structure. Cry1Aa, Cry3Aa, and Cry11Bb contain the most variable regions in the loops of domain II, which determine the specificity of these toxins. This is the first model of a Cry 11 protein and its importance can be perceived since members of this group of toxins are potentially important candidates for mosquito control programs. The coordinates of the Cry $11 \mathrm{Bb}$ model can be obtained upon request to sorduz@epm.net.co.

\section{REFERENCES}

Aronson A, Geng C, Wu L 1999. Aggregation of Bacillus thuringiensis Cry1A toxin upon binding to target insect larval midgut vesicles. Appl Environm Microbiol 65: 2503-2507.
Bosch D, Schipper B, Van der Kleij H, de Maagd RA, Stiekema WJ 1994. Recombinant Bacillus thuringiensis crystal proteins with new properties: possibilities for resistant management. Biotechnology 12: 915-919.

Chen XJ, Lee MK, Dean DH 1993. Site-directed mutations in a highly conserved region of Bacillus thurngiensis $\partial$-endotoxin affect inhibition of short circuit current across Bombyx mori midguts. Proc Natl Acad Sci USA 90: 9041-9045.

Crickmore N, Zeigler DR, Feitelson J, Schnepf E, Van Rie J, Lereclus D, Baum J, Dean DH 1998. Revision of the nomenclature for the Bacillus thuringiensis pesticidal crystal proteins. Microbiol Mol Biol Rev 62: 807-813.

Dai S-M, Gill S 1993. In vitro and in vivo proteolysis of the Bacillus thuringiensis subsp. israelensis CryIVD protein by Culex quinquefasciatus larval midgut proteases. Insect Biochem Molec Biol 23: 273-283.

Feldmann F, Dullemans A, Waalwijk C 1995. Binding of the CryIVD toxin of Bacillus thuringiensis to larval dipteran midgut proteins. Appl Environ Microbiol 61: 2601-2605.

Gazit E, Shai Y 1993. Structural and functional organization of the $\alpha 5$ segment of Bacillus thuringiensis endotoxins. Biochemistry 32: 3429-3436.

Gazit E, La Rocca P, Sansom MSP, Shai Y 1998. The structure and organization within the membrane of the helices composing the pore forming domain of Bacillus thuringiensis $\partial$-endotoxin are consistent with an "umbrella-like" structure of the pore. Proc Natl Acad Sci USA 95: 12289-12294.

Ge AZ, Sivarova ND, Dean DH 1989. Location of the Bombyx mori specificity domain of Bacillus thuringiensis d-endotoxin protein. Proc Natl Acad Sci USA 86: 4037-4041.

Gill SS, Cowless EA, Pietrantonio PV 1992. The mode of action of Bacillus thuringiensis endotoxins. Annu Rev Entomol 37: 615-636.

Grochulski P, Masson L, Borisova S, Pusztai-Carey M, Schwartz JL, Brousseau R, Cygler M 1995. Bacillus thuringiensis CryIA(a) insecticidal toxin: crystal structure and channel formation. J Mol Biol 254: 447-464. 
Guex N, Peitsch MC 1997. SWISS-MODEL and the Swiss-PdbViewer: an environment for comparative protein modeling. Electrophoresis 18: 2714-2723.

Hofmann C, Vanderbruggen H, Hofte H, Van Mellaert H 1988. Specificity of Bacillus thuringiensis deltaendotoxins is correlated with the presence of high affinity binding sites in the brushborder membrane of the insect membrane of target insect midguts. Proc Natl Acad Sci USA 85: 7844-7848.

Knowles BH 1994. Mechanism of action of Bacillus thuringiensis insecticidal $\partial$-endotoxins. Advan Insect Physiol 24: 275-308.

Knowles BH, Ellar DJ 1987. Colloid osmotic lysis is a general feature of the mechanism of action of Bacillus thuringiensis delta-endotoxins with different insect specificities. Biochim Biophys Acta 924: 509-518.

Kumar ASM, Aronson AI 1999. Analysis of mutations in the pore-forming region essential for insecticidal activity of a Bacillus thuringiensis $\partial$-endotoxin. $J$ Bacteriol 181: 6103-6107.

Laskowski RA, MacArthur MW, Moss DS, Thornton JM 1993. PROCHECK: a program to check the stereochemical quality of protein structures. $J$ Appl Cryst 26: 283-291.

Lee MK, You TH, Gould FL, Dean DH 1999. Identification of residues in domain III of Bacillus thuringiensis Cry1Ac toxin that affect binding and toxicity. Appl Environ Microbiol 65: 4513-4520.

Li J, Carroll J, Ellar DJ 1991. Crystal structure of insecticidal $\partial$-endotoxin from Bacillus thuringiensis at 2.5 A resolution. Nature 353: 815-821.

Lu H, Rajamohan F, Dean DH 1994. Identification of amino acid residues of Bacillus thuringiensis $\partial$-endotoxin CryIA(a) associated with membrane binding and toxicity to Bombyx mori. J Bacteriol 176: 5554-5559.

Masson L, Mazza A, Gringorten JL, Baines D, Anelunias V, Brousseau R 1994. Specificity domain localization of Bacillus thuringiensis insecticidal CryIA toxins is highly dependent on the bioassay system. Mol Microbiol 14: 851-860.

Orduz S, Diaz T, Thiéry I, Charles J-F, Rojas W 1994. Crystal proteins from Bacillus thuringiensis subspecies medellin. Appl Microbiol Biotechnol 40: 794799.

Orduz S, Realpe M, Arango R, Murillo LA, Delecluse A 1998. Sequence of the cry11Bb11 gene from $B a$ cillus thuringiensis subsp. medellin and toxicity analysis of its encoded protein. Biochim Biophys Acta 1388: 267-272.

Orduz S, Rojas W, Correa MM, Montoya AE, de Barjac H 1992. A new serotype of Bacillus thuringiensis from Colombia toxic to mosquito larvae. J Invertebr Pathol 59: 99-103.

Ragni A, Thiery I, Delecluse A 1996. Characterization of six highly mosquitocidal Bacillus thuringiensis strains that do not belong to $\mathrm{H}-14$ serotype. Curr Microbiol 32: 48-54.

Schnepf E, Crickmore N, van Rie J, Lereclus D, Baum J, Feitelson J, Zeigler DR, Dean DH 1998. Bacillus thuringiensis and its pesticidal crystal proteins. Microbiol Mol Biol Rev 62: 772-806.

Schnepf E, Tomczak K, Ortega JP, Whiteley HR 1990. Specificity-determining regions of lepidopteran-specific insecticidal proteins produced by Bacillus thuringiensis. J Biol Chem 265: 20923-20930.

Schwartz JL, Potvin L, Chen XJ, Brousseau R, Laprade R, Dean DH 1997. Single-site mutations in the conserved alternating-arginine region affect ion channels formed by CryIAa, a Bacillus thuringiensis toxin. Appl Environ Microbiol 63: 3978-3984.

Segura C, Guzman F, Patarroyo ME, Orduz S 2000. Activation pattern and toxicity of the Cry $11 \mathrm{Bb}$ toxin of Bacillus thuringiensis subsp. medellin. J Invertebr Pathol 76: 56-62.

Smith GP, Ellar DJ 1994. Mutagenesis of two surface exposed loops of the Bacillus thuringiensis CryIC $\partial$-endotoxin affects insecticidal specificity. Biochem J 302: 611-616.

Thiery I, Hamon S, Delecluse A, Orduz S 1998. The introduction into Bacillus sphaericus of the Bacillus thuringiensis subsp. medellin $\mathrm{Cyt} 1 \mathrm{Ab} 1$ gene results in higher susceptibility of resistant mosquito larva population to B. sphaericus. Appl Environ Microbiol 64: 3910-3916.

Vriend G 1990. WHAT IF: a molecular modeling and drug design program. J Mol Graph 8: 52-56.

von Tersch MA, Slatin SL, Kuleszca CA, English LH 1994. Membrane-permeabilizing activities of Bacillus thuringiensis coleopteran-active toxin CryI-IIB2 and CryIIIB2 domain I peptide. Appl Environ Microbiol 60: 3711-3717.

Walters FS, Slatin SL, Kuleszca CA, English LH 1993. Ion Channel activity of $\mathrm{N}$-terminal fragments from CryIA(c) d-endotoxin. Biochem Biophys Res Commun 196: 921-926. 